\title{
A revised nomenclature for transcribed human endogenous retroviral loci
}

Jens Mayer ${ }^{1}$, Jonas Blomberg ${ }^{2}$ and Ruth $L$ Seal $^{\beta^{*}}$

\begin{abstract}
Background: Endogenous retroviruses (ERVs) and ERV-like sequences comprise $8 \%$ of the human genome. A hitherto unknown proportion of ERV loci are transcribed and thus contribute to the human transcriptome. A small proportion of these loci encode functional proteins. As the role of ERVs in normal and diseased biological processes is not yet established, transcribed ERV loci are of particular interest. As more transcribed ERV loci are likely to be identified in the near future, the development of a systematic nomenclature is important to ensure that all information on each locus can be easily retrieved.
\end{abstract}

Results: Here we present a revised nomenclature of transcribed human endogenous retroviral loci that sorts loci into groups based on Repbase classifications. Each symbol is of the format ERV + group symbol + unique number. Group symbols are based on a mixture of Repbase designations and well-supported symbols used in the literature. The presented guidelines will allow newly identified loci to be easily incorporated into the scheme.

Conclusions: The naming system will be employed by the HUGO Gene Nomenclature Committee for naming transcribed human ERV loci. We hope that the system will contribute to clarifying a certain aspect of a sometimes confusing nomenclature for human endogenous retroviruses. The presented system may also be employed for naming transcribed loci of human non-ERV repeat loci.

\section{Human endogenous retroviruses}

Human endogenous retroviruses (ERVs) are remnants of infections of former exogenous retroviruses. Proviruses formed by numerous distinct exogenous retroviruses in the germline genome could be inherited by subsequent generations. About $8 \%$ of the human genome consists of sequences that are potentially of retroviral origin [1] and are distributed in about 700,000 different loci. In addition to proviruses, these sequences include solitary long terminal repeats (LTRs), nonretroviral sequences flanked by LTRs that may not be directly derived from infectious retroviruses and sequences similar to LTRs. ERVs and related sequences are thus part of the repetitive portions of the human genome, which comprise about $45 \%$ of the human genome mass, including mobile DNA such as L1, Alu and SVA elements.

\footnotetext{
* Correspondence: rseal@ebi.ac.uk

${ }^{3} \mathrm{HUGO}$ Gene Nomenclature Committee, European Molecular Biology Laboratory-European Bioinformatics Institute, Wellcome Trust Genome Campus, Hinxton, Cambridgeshire CB10 1SD, UK Full list of author information is available at the end of the article
}

Detailed analysis of the human genome sequence by wet-lab and bioinformatics approaches resulted in the definition of ERV groups, with the number depending on the methods used for defining groups: 31 groups were defined by Sperber et al. [2] and Blomberg et al. [3], 42 groups were defined by Mager and Medstrand [4], 30 groups were defined by Gifford and Tristem [5] and several hundred human ERV and LTR families were defined by Repbase [6].

Almost all human ERV loci no longer encode former retroviral proteins because of their ancient incorporation into the host genome and thus accumulation of nonsense mutations. Many loci are missing large proviral portions, and most loci have been reduced to so-called solitary LTRs by homologous recombination between proviral LTRs. For more detailed information on human ERVs, we refer interested readers to recent reviews on the topic and the references therein [7-10].

While protein coding capacity is very limited, many human ERV loci still are transcribed and usually are initiated by promoter sequences within the proviral LTRs. Obviously, mutations within LTRs have not yet
C Biomed Central 
rendered all LTRs in the human genome defective. In principle, promoters in flanking, non-ERV sequences may also contribute to transcription of those loci. Probably every human tissue and cell type, diseased or not, contains ERV transcripts [11,12]. More than a single ERV group is usually found transcribed, and patterns of transcribed ERV groups differ between tissue and cell types. Transcription of ERV loci is thus regulated in some way. While expression of ERV sequences has been associated with a number of human diseases, such as germ cell tumours, melanoma and multiple sclerosis, the involvement of ERVs in human diseases remains to be elucidated. On the other side, some ERV loci very likely provide important biological functions, such as the syncytin [13] and syncytin 2 loci [14], referred to herein as ERVW-1 and ERVFRD-1, respectively. Other loci harbouring only partial open reading frames, such as a recently characterized HERV-W locus on chromosome Xq22.3 [15] (ERVW-2), may likewise produce partial retroviral proteins with potential biological functions. It is therefore of particular interest which ERV loci actually contribute to the human transcriptome.

Recent studies have identified transcribed ERV loci in normal and diseased human cells and tissues by means of reassigning ERV cDNA sequences to individual loci in the human reference genome sequence, employing characteristic nucleotide differences between individual loci of a regarded ERV group. Many more transcribed ERV loci are likely to be identified in future studies. It is therefore necessary to introduce a nomenclature for transcribed human ERV sequences.

\section{Previous nomenclature used in the literature}

The lack of an established nomenclature for transcribed ERV elements has led to confusion within the literature. These problems were previously reviewed in detail [16]. ERVs have been classified into groups (formerly known as "families", which is heresy to virologists because "family" refers to Retroviridae), although different classification systems have been used. For instance, some groups have been defined initially by molecular genetics means, others by sequence similarity and others by primer binding site sequences. Changing amounts of sequence information also showed that some ERV groups' designations needed to be revised. Different names have been used for the same ERV group. Likewise, individual loci have been referred to using a variety of different symbols (for example, see the aliases listed in Table 1 for the ERVK-6 locus). The use of different symbols for the same locus makes it difficult to retrieve all information on that particular locus.

\section{Previous ERV nomenclature and the Human Genome Organisation Gene Nomenclature Committee}

The Human Genome Organisation (HUGO) Gene Nomenclature Committee (HGNC) works under the auspices of HUGO and is the only worldwide authority that assigns standardised nomenclature to human genes [17]. The HGNC has previously focused on approving nomenclature for protein-coding genes, pseudogenes, phenotypes and noncoding RNA. In the past, the committee has approved symbols for specific human ERVs only at the request of individual researchers. The symbols did not follow a systematic nomenclature: some symbols were of a simple format (for example, ERV1), some provided information on the group to which the ERV belonged (for example, ERVK2) and others included information on proteins encoded by the ERV (for example, ERVWE1 (endogenous retroviral family $\mathrm{W}$, env $(\mathrm{C} 7)$, member 1$)$ ). On reviewing the literature, it was clear that (1) many of the most frequently published loci were not represented by HGNC symbols, (2) by following more than one system, HGNC symbols were not serving the community, and (3) the nomenclature needed both updating and expansion.

HGNC editors curate relevant information for each gene that has approved nomenclature. In addition to approving a gene symbol and name for each transcribed human ERV, the HGNC records all known symbol aliases so that information on each gene can be retrieved using any known symbol. HGNC entries also include the chromosomal location of the ERV locus, links to GenBank, European Molecular Biology Laboratory (EMBL) and DNA Databank of Japan (DDBJ) sequence records and links to at least one PubMed reference. Where appropriate, links are also provided to annotation projects at both the genomic and proteomic levels. HGNC names are propagated to other major biological databases, such as Ensembl, UniProt and Entrez Gene. Therefore, this new nomenclature will provide a useful resource that is currently unavailable to the ERV community and other researchers concerned with ERVs.

\section{A gene-based nomenclature}

The primary definition of a gene used by the HGNC is "a DNA segment that contributes to phenotype/function" [18]. It is beyond the scope of this nomenclature effort to standardise the nomenclature of ERVs in general or to attempt to name every ERV element in the genome. As discussed above, there is evidence that some human ERVs encode functional proteins and that some encode transcripts and/or proteins which may be associated with disease, so the transcriptionally active loci come under the remit of the HGNC for naming. 
Table 1 Nomenclature for transcribed human endogenous retrovirus loci

\begin{tabular}{|c|c|c|c|c|c|}
\hline Symbol & Name & $\begin{array}{l}\text { Chromosome } \\
\text { location }\end{array}$ & Aliases & $\begin{array}{l}\text { Representative GenBank } \\
\text { accession numbers }\end{array}$ & Sources \\
\hline ERVK-1 $1^{a}$ & $\begin{array}{l}\text { endogenous retrovirus group } \mathrm{K}, \\
\text { member } 1\end{array}$ & $1 \mathrm{p} 31.1$ & C1_A & FN806826, BQ304053 & $\begin{array}{l}{[23]} \\
{[24]}\end{array}$ \\
\hline ERVK-2 & $\begin{array}{l}\text { endogenous retrovirus group } \mathrm{K}, \\
\text { member } 2\end{array}$ & $3 p 25.3$ & C3_A & FN806829, EF153331 & [23] \\
\hline ERVK-3 & $\begin{array}{l}\text { endogenous retrovirus group } \mathrm{K}, \\
\text { member } 3\end{array}$ & $3 q 13.2$ & C3_B & FN806830 & [23] \\
\hline ERVK-4 & $\begin{array}{l}\text { endogenous retrovirus group } \mathrm{K} \text {, } \\
\text { member } 4\end{array}$ & $3 q 21.2$ & C3_C, ERVK4, HERV-K(I) & FN806831 & $\begin{array}{l}{[23]} \\
{[25]}\end{array}$ \\
\hline ERVK-5 & $\begin{array}{l}\text { endogenous retrovirus group } \mathrm{K}, \\
\text { member } 5\end{array}$ & $3 q 12.3$ & ERVK5, HERV-K(II) & CF227259, AK021866 & $\begin{array}{l}{[24]} \\
{[25]}\end{array}$ \\
\hline ERVK-6 & $\begin{array}{l}\text { endogenous retrovirus group } \mathrm{K}, \\
\text { member } 6\end{array}$ & $7 p 22.1$ & $\begin{array}{l}\text { C7_A, ERVK6, HERV-K108, HERV-K(HML-2. } \\
\text { HOM), envK2, HERV-K(C7) }\end{array}$ & $\begin{array}{l}\text { FN806837, AY371029, X82271, } \\
\text { AF080233 }\end{array}$ & $\begin{array}{l}{[23]} \\
{[26]} \\
{[27]}\end{array}$ \\
\hline ERVK-7 & $\begin{array}{l}\text { endogenous retrovirus group } \mathrm{K}, \\
\text { member } 7\end{array}$ & $1 q 22$ & C1_B, ERVK7, HERV-K102 & $\begin{array}{l}\text { FN806827, EF153338, S46404, } \\
\text { DQ069911 }\end{array}$ & $\begin{array}{l}{[23]} \\
{[28]}\end{array}$ \\
\hline ERVK-8 & $\begin{array}{l}\text { endogenous retrovirus group } \mathrm{K} \text {, } \\
\text { member } 8\end{array}$ & $8 p 23.1$ & C8_A, ERVK8, HERV-K115, envK6 & FN806840 & $\begin{array}{l}{[23]} \\
{[27]} \\
{[29]}\end{array}$ \\
\hline ERVK-9 & $\begin{array}{l}\text { endogenous retrovirus group } \mathrm{K} \text {, } \\
\text { member } 9\end{array}$ & $6 q 14.1$ & c6_A, HERV-K109, envK4 & $\begin{array}{l}\text { FN806836, AF080234, } \\
\text { AY371030 }\end{array}$ & $\begin{array}{l}{[23]} \\
{[26]} \\
{[27]} \\
{[28]}\end{array}$ \\
\hline ERVK-10 & $\begin{array}{l}\text { endogenous retrovirus group } \mathrm{K} \text {, } \\
\text { member } 10\end{array}$ & $5 q 33.3$ & C5_A, HERV-K10 & $\begin{array}{l}\text { FN806835, AF080231, } \\
\text { CN345079 }\end{array}$ & $\begin{array}{l}{[23]} \\
{[24]} \\
{[26]}\end{array}$ \\
\hline ERVK-11 & $\begin{array}{l}\text { endogenous retrovirus group } \mathrm{K}, \\
\text { member } 11\end{array}$ & $3 q 27.2$ & C3_E, N8.4, HML-2A & $\begin{array}{l}\text { FN806833, AF080232, } \\
\text { AF080229, U87590 }\end{array}$ & {$[23,26]$} \\
\hline ERVK-12 & $\begin{array}{l}\text { endogenous retrovirus group } \mathrm{K}, \\
\text { member } 12\end{array}$ & $4 q 32.3$ & C4_A & FN806834, EF153341 & [23] \\
\hline ERVK-13 & $\begin{array}{l}\text { endogenous retrovirus group } \mathrm{K}, \\
\text { member } 13\end{array}$ & $3 q 24$ & C3_D & FN806832 & [23] \\
\hline ERVK-14 & $\begin{array}{l}\text { endogenous retrovirus group } \mathrm{K}, \\
\text { member } 14\end{array}$ & $7 q 22.1$ & C7_B & FN806838 & [23] \\
\hline ERVK-15 & $\begin{array}{l}\text { endogenous retrovirus group } \mathrm{K}, \\
\text { member } 15\end{array}$ & $7 q 34$ & C7_C, P1.10 & FN806839, U87594 & [23] \\
\hline ERVK-16 & $\begin{array}{l}\text { endogenous retrovirus group } \mathrm{K}, \\
\text { member } 16\end{array}$ & 10p14 & C10_A. M3.8 & FN806841, EF543114, U87587 & $\begin{array}{l}{[23]} \\
{[27]} \\
{[30]}\end{array}$ \\
\hline ERVK-17 & $\begin{array}{l}\text { endogenous retrovirus group } \mathrm{K}, \\
\text { member } 17\end{array}$ & $10 q 24.2$ & C10_B & FN806842, AF080230 & $\begin{array}{l}{[23]} \\
{[26]}\end{array}$ \\
\hline ERVK-18 & $\begin{array}{l}\text { endogenous retrovirus group } \mathrm{K} \text {, } \\
\text { member } 18\end{array}$ & $1 \mathrm{q} 23.3$ & C1_C, HERV-K18 & FN806828 & $\begin{array}{l}{[23]} \\
{[31]}\end{array}$ \\
\hline ERVK-19 & $\begin{array}{l}\text { endogenous retrovirus group } \mathrm{K}, \\
\text { member } 19\end{array}$ & $19 q 11$ & P1.8, HERV-K(C19), envK3 & U87593 & $\begin{array}{l}{[27,30]} \\
{[31]}\end{array}$ \\
\hline ERVK-20 & $\begin{array}{l}\text { endogenous retrovirus group } \mathrm{K}, \\
\text { member } 20\end{array}$ & $11 q 23.3$ & C11_B & FN806844 & [23] \\
\hline ERVK-21 & $\begin{array}{l}\text { endogenous retrovirus group K, } \\
\text { member } 21\end{array}$ & $12 q 14.1$ & C12_A, envK1 & FN806845, U32496 & $\begin{array}{l}{[23]} \\
{[27]}\end{array}$ \\
\hline ERVK-22 & $\begin{array}{l}\text { endogenous retrovirus group } \mathrm{K}, \\
\text { member } 22\end{array}$ & 19p13.3 & C19_A & FN806846, EF153351 & [23] \\
\hline ERVK-23 & $\begin{array}{l}\text { endogenous retrovirus group } \mathrm{K} \text {, } \\
\text { member } 23\end{array}$ & $21 \mathrm{q} 21.1$ & C21_A & FN806847, EF543113 & [23] \\
\hline ERVK-24 & $\begin{array}{l}\text { endogenous retrovirus group } \mathrm{K}, \\
\text { member } 24\end{array}$ & $22 q 11.21$ & C22_A, HERV-K101 & $\begin{array}{l}\text { FN806848, AU124350, } \\
\text { AA580921, AW812040 }\end{array}$ & $\begin{array}{l}{[23]} \\
{[28]}\end{array}$ \\
\hline ERVK-25 & $\begin{array}{l}\text { endogenous retrovirus group } \mathrm{K}, \\
\text { member } 25\end{array}$ & $11 q 22.1$ & C11_A & $\begin{array}{l}\text { FN806843, CF227268, } \\
\text { AW818206 }\end{array}$ & [23] \\
\hline
\end{tabular}


Table 1 Nomenclature for transcribed human endogenous retrovirus loci (Continued)

\begin{tabular}{|c|c|c|c|c|c|}
\hline$\overline{E R V K 3-1^{b}}$ & $\begin{array}{l}\text { endogenous retrovirus group } \mathrm{K} 3 \text {, } \\
\text { member } 1\end{array}$ & $19 q 13.43$ & HERV-K(HML6-1) & $\begin{array}{l}\text { AK054868, BC010118, } \\
\text { BC011670 }\end{array}$ & [32] \\
\hline ERVK3-2 & $\begin{array}{l}\text { endogenous retrovirus group } \mathrm{K} 3 \text {, } \\
\text { member } 2\end{array}$ & $14 q 24.2$ & HML6-c14 & $\begin{array}{l}\text { AK027828, AK096726, } \\
\text { CR591084 }\end{array}$ & [32] \\
\hline ERVK3-3 & $\begin{array}{l}\text { endogenous retrovirus group } \mathrm{K} 3 \text {, } \\
\text { member } 3\end{array}$ & $5 q 13.2$ & HML6-c5 & FR714893 & [33] \\
\hline ERVK3-4 & $\begin{array}{l}\text { endogenous retrovirus group } \mathrm{K} 3 \text {, } \\
\text { member } 4\end{array}$ & $11 \mathrm{p} 15.4$ & HML6-c11 & FR714894 & [33] \\
\hline ERVK3-5 & $\begin{array}{l}\text { endogenous retrovirus group } \mathrm{K} 3 \text {, } \\
\text { member } 5\end{array}$ & $12 q 24.12$ & HML6-c12 & FR714895 & [33] \\
\hline ERVK3-6 & $\begin{array}{l}\text { endogenous retrovirus group } \mathrm{K} 3 \text {, } \\
\text { member } 6\end{array}$ & $19 q 13.41$ & HML6-C19A & FR714896 & [33] \\
\hline ERVK3-7 & $\begin{array}{l}\text { endogenous retrovirus group } \mathrm{K} 3 \text {, } \\
\text { member } 7\end{array}$ & 19p13.2 & HML6-C19B & FR714897 & [33] \\
\hline ERVK3-8 & $\begin{array}{l}\text { endogenous retrovirus group } \mathrm{K} 3 \text {, } \\
\text { member } 8\end{array}$ & 20p11.21 & HML6-c20 & FR714898 & [33] \\
\hline ERVFRD-1 & $\begin{array}{l}\text { endogenous retrovirus group } \\
\text { FRD, member } 1\end{array}$ & $6 \mathrm{p} 24.2$ & HERV-FRD, envFRD, ERVFRDE1, syncytin 2 & $\begin{array}{l}\text { AK075092, AK123938, } \\
\text { AY358244 }\end{array}$ & $\begin{array}{l}{[24]} \\
{[27]} \\
{[34]}\end{array}$ \\
\hline ERVFRD- $2^{c}$ & $\begin{array}{l}\text { endogenous retrovirus group } \\
\text { FRD, member } 2\end{array}$ & $19 q 13.41$ & FL445949 & AK127846 & \\
\hline ERV3-1 & $\begin{array}{l}\text { endogenous retrovirus group } 3 \text {, } \\
\text { member } 1\end{array}$ & $7 q 11.21$ & ERV3, envR & AK295189 & $\begin{array}{l}{[24]} \\
{[27]} \\
{[35]} \\
{[36]}\end{array}$ \\
\hline$\overline{E R V 3-2^{c}}$ & $\begin{array}{l}\text { endogenous retrovirus group 3, } \\
\text { member } 2\end{array}$ & $7 q 33$ & KIAA1466 & AB040899, AL833192 & \\
\hline $\begin{array}{l}\text { ERVPABLB- } \\
1\end{array}$ & $\begin{array}{l}\text { endogenous retrovirus group } \\
\text { PABLB, member } 1\end{array}$ & $3 p 24.3$ & envR(b) & $\begin{array}{l}\text { BQ012865, CF529244, } \\
\text { Al189490 }\end{array}$ & {$[27]$} \\
\hline ERVFC1-1 & $\begin{array}{l}\text { endogenous retrovirus group } \\
\text { FC1, member } 1\end{array}$ & $7 q 36.2$ & envF(c)2 & AK124285 & {$[27]$} \\
\hline ERWW-1 & $\begin{array}{l}\text { endogenous retrovirus group W, } \\
\text { member } 1\end{array}$ & $7 q 21.2$ & $\begin{array}{l}\text { ERVWE1, syncytin 1, enverin, envW, HERV- } \\
\text { W-ENV, HERV-7q, HERV7Q }\end{array}$ & $\begin{array}{l}\text { BG012022, AF208161, } \\
\text { BX391741, BX365066 }\end{array}$ & $\begin{array}{l}{[13,24]} \\
{[27]} \\
{[37]}\end{array}$ \\
\hline ERVW-2 & $\begin{array}{l}\text { endogenous retrovirus group W, } \\
\text { member } 2\end{array}$ & $\mathrm{Xq} 22.3$ & ERWWE2, Penv-C15 & AF127228, FN689795 & [38] \\
\hline ERVW-3 & $\begin{array}{l}\text { endogenous retrovirus group W, } \\
\text { member } 3\end{array}$ & $3 q 23$ & CL4 & AF127227 & [39] \\
\hline ERVW-4 & $\begin{array}{l}\text { endogenous retrovirus group W, } \\
\text { member } 4\end{array}$ & $15 q 21.3$ & C187-23 & AF123882, EF539878 & $\begin{array}{l}{[38]} \\
{[39]} \\
{[40]} \\
\end{array}$ \\
\hline ERVW-5 & $\begin{array}{l}\text { endogenous retrovirus group W, } \\
\text { member } 5\end{array}$ & $3 q 26.32$ & $\mathrm{CL} 2$ & AF123881 & [39] \\
\hline ERVW-6 & $\begin{array}{l}\text { endogenous retrovirus group W, } \\
\text { member } 6\end{array}$ & $11 q 14.1$ & & AK022306, AB063619 & [41] \\
\hline ERVS71-1 & $\begin{array}{l}\text { endogenous retrovirus group } \\
\text { S71, member } 1\end{array}$ & 19p13.11 & envT & $\begin{array}{l}\text { CN288807, BQ932595, } \\
\text { BQ941761 }\end{array}$ & $\begin{array}{l}{[27]} \\
{[42]}\end{array}$ \\
\hline ERVS71-2 & $\begin{array}{l}\text { endogenous retrovirus group } \\
\mathrm{S71} \text {, member } 2\end{array}$ & 10p11.1 & HERV-HC2 & AB167270, AB167277 & [43] \\
\hline ERVFH21-1 & $\begin{array}{l}\text { endogenous retrovirus group } \\
\mathrm{FH} 21 \text {, member } 1\end{array}$ & $7 p 21.3$ & HERV-F(XA34) & AK023847 & [32] \\
\hline ERVH48-1 & $\begin{array}{l}\text { endogenous retrovirus group } 48 \text {, } \\
\text { member } 1\end{array}$ & $21 q 22.3$ & C21orf105, HERV-F (type b) & BC005107, CR591419 & {$[32]$} \\
\hline ERVE-1 & $\begin{array}{l}\text { endogenous retrovirus group } E_{\text {, }} \\
\text { member } 1\end{array}$ & $17 q 11.2$ & ERVE1 & BC037342, FM212572 & [44] \\
\hline ERVE-2 & $\begin{array}{l}\text { endogenous retrovirus group } E_{\text {, }} \\
\text { member } 2\end{array}$ & $11 q 13.2$ & & FM212575 & [45] \\
\hline
\end{tabular}


Table 1 Nomenclature for transcribed human endogenous retrovirus loci (Continued)

\begin{tabular}{|c|c|c|c|c|c|}
\hline ERVE-3 & $\begin{array}{l}\text { endogenous retrovirus group } E_{\text {, }} \\
\text { member } 3\end{array}$ & $8 p 23.1$ & & FM212573 & [45] \\
\hline ERVE-4 & $\begin{array}{l}\text { endogenous retrovirus group } \mathrm{E} \text {, } \\
\text { member } 4\end{array}$ & $6 q 15$ & CT-RCC-1 & EU137846 & [46] \\
\hline ERW-1 & $\begin{array}{l}\text { endogenous retrovirus group } V \text {, } \\
\text { member } 1\end{array}$ & $19 q 13.41$ & HERV-V1, ENW1 & $\begin{array}{l}\text { AK056776, BC104018, } \\
\text { BC104019 }\end{array}$ & [47] \\
\hline ERW-2 & $\begin{array}{l}\text { endogenous retrovirus group } \mathrm{V} \text {, } \\
\text { member } 2\end{array}$ & $19 q 13.41$ & HERV-V2, ENW2 & $\begin{array}{l}\text { Al434519, CA417098, } \\
\text { DA863698 }\end{array}$ & {$[47]$} \\
\hline ERVI-1 ${ }^{c}$ & $\begin{array}{l}\text { endogenous retrovirus group I, } \\
\text { member } 1\end{array}$ & $9 q 22.1$ & & $\begin{array}{l}\text { AK124340, AK124077, } \\
\text { CR614956 }\end{array}$ & \\
\hline$\overline{E R V 18-1^{c}}$ & $\begin{array}{l}\text { endogenous retrovirus group } 18 \text {, } \\
\text { member } 1\end{array}$ & $15 q 21.3$ & & AK126787 & \\
\hline $\begin{array}{l}\text { ERVMER61- } \\
1^{c}\end{array}$ & $\begin{array}{l}\text { endogenous retrovirus group } \\
\text { MER61, member } 1\end{array}$ & $1 \mathrm{q} 31.3$ & C1orf99 & BC040856 & \\
\hline ERVH-1 & $\begin{array}{l}\text { endogenous retrovirus group } \mathrm{H} \text {, } \\
\text { member } 1\end{array}$ & $4 \mathrm{p} 15.2$ & HERV-H4p15.2 & EU669866, BC015108 & [48] \\
\hline ERVH-2 & $\begin{array}{l}\text { endogenous retrovirus group } \mathrm{H} \text {, } \\
\text { member } 2\end{array}$ & Xp22.32 & HERV-HX & EU195218, EU195219 & {$[48]$} \\
\hline ERVH-3 & $\begin{array}{l}\text { endogenous retrovirus group } \mathrm{H} \text {, } \\
\text { member } 3\end{array}$ & $6 q 12$ & $\mathrm{HERV}-\mathrm{H} / \mathrm{F}$ & AJ431196 & [49] \\
\hline ERVH-4 & $\begin{array}{l}\text { endogenous retrovirus group } \mathrm{H} \text {, } \\
\text { member } 4\end{array}$ & $14 q 32.2$ & clone c4.2 & U35031, U88895 & {$[50]$} \\
\hline ERVH-5 & $\begin{array}{l}\text { endogenous retrovirus group } \mathrm{H} \text {, } \\
\text { member } 5\end{array}$ & 10p12.1 & clone c14.6 & U35033 & {$[50]$} \\
\hline ERVH-6 & $\begin{array}{l}\text { endogenous retrovirus group } \mathrm{H} \text {, } \\
\text { member } 6\end{array}$ & Yq11.223 & & U88898 & {$[50]$} \\
\hline ERVH-7 & $\begin{array}{l}\text { endogenous retrovirus group } \mathrm{H} \text {, } \\
\text { member } 7\end{array}$ & $14 q 32.12$ & & BC039675, T67812 & {$[50]$} \\
\hline ERV9-1 & $\begin{array}{l}\text { endogenous retrovirus group } 9 \text {, } \\
\text { member } 1\end{array}$ & $11 q 13.2$ & pTR2 & X15673, X15675, X57147 & $\begin{array}{l}{[51]} \\
{[52]}\end{array}$ \\
\hline
\end{tabular}

${ }^{a}$ The ERVK group is referred to as HERV-K(HML-2) according to a nomenclature introduced by Andersson et al. in 1999 [53]. ${ }^{\mathrm{b}}$ The ERVK3 group has been referred to as HERV-K(HML-6) according to the Andersson et al. classification scheme [53]. 'The following loci have not appeared in publications but have been annotated by the RefSeq project: ERVFRD-2 (Entrez Gene:388560); ERVI-1 (Entrez Gene ID:100131068); ERV18-1 (Entrez Gene ID:100133791); ERVMER61-1 (Entrez Gene ID:339476).

This category of ERVs represents most of the individual loci that have been published with individual names, so it is worth developing a standardised nomenclature for this subset. The three criteria for being accepted as a transcriptionally active ERV are as follows: (1) The ERV must be represented by an mRNA sequence in a public database, (2) the reported cDNA sequence must map unambiguously to the reference genome to allow identification and (3) the sequence must represent a viral gene rather than solely a solitary LTR. We acknowledge that there are sources of uncertainty. Many ERVs may be expressed at a low level [19], a "leakage" which can be hard to distinguish from perhaps more significant expression. Groups of recently integrated ERVs may be highly expressed, but their transcripts may be identical or almost identical and could be hard to map unambiguously. However, these difficulties should not prevent the naming of ERV loci which fulfil the criteria mentioned above. There is one symbol approved per ERV locus independently of how many viral genes the ERV may encode.

\section{A systematic ERV nomenclature scheme}

The nomenclature scheme described in this paper aims to be concise so that it is user-friendly. It also aims to be informative to researchers, including those who are less familiar with the field. To be informative, the nomenclature scheme is hierarchical, with each symbol beginning with the root symbol "ERV" so that the symbols are instantly recognisable and can be grouped together in searches. Note that many researchers have published papers using symbols beginning with "HERV", but it is against the guidelines of the HGNC ever to use $\mathrm{H}$ for "human" in symbols, mainly because this precludes the possibility of the nomenclature scheme's being extended to other species. Each ERV symbol, then, includes an identifier that represents the group to which the ERV belongs. 
In order for the nomenclature scheme to be systematic, one method of sorting ERVs into groups needed to be selected. The Repbase system [6] is a widely known, comprehensive database of repetitive elements that groups ERVs together on the basis of sequence similarity. RepeatMasker annotations using Repbase designations are available on the University of California, Santa Cruz (UCSC) [20], and Ensembl [21] genome browsers, making these ERV groups highly accessible and recognisable to researchers in the field. Therefore, the nomenclature system uses the Repbase classification system for naming the ERVs within groups. Repbase groups, however, do not follow a systematic nomenclature and often contain an unallowable " $\mathrm{H}$ " for "human". When deciding on the group identifier to be included in each symbol, we compared Repbase symbols with those that have appeared frequently in the literature. In cases where there was a well-supported nomenclature present in the literature, we used this symbol in place of the Repbase symbol; for example, we used ERVW instead of the Repbase group designation HERV17, as we felt that these would be more likely to be used by the ERV community. For a comparison of the group symbols used in the new nomenclature scheme with Repbase designations, see Table 2.

Finally, each ERV within a particular group is uniquely identified by a number, for example, ERVK-1. Numbers are assigned consecutively within each group to make the nomenclature system expandable. The number is used to make each symbol unique and has no intrinsic meaning. ERVK-2 has merely been assigned the next number following ERVK-1, but this provides no

\begin{tabular}{|c|c|}
\hline $\begin{array}{l}\text { Repbase group } \\
\text { symbol }\end{array}$ & $\begin{array}{l}\text { Group symbol in new nomenclature } \\
\text { scheme }\end{array}$ \\
\hline HERVK & ERVK \\
\hline HERVK3 & ERVK3 \\
\hline MER50 & ERVFRD \\
\hline ERV3 & ERV3 \\
\hline PABL_B & ERVPABLB \\
\hline HERV-FC1 & ERVFC1 \\
\hline HERV17 & ERWW \\
\hline HERVS71 & ERVS71 \\
\hline HERVH48 & ERVH48 \\
\hline HERVFH21 & ERVFH21 \\
\hline HERVE & ERVE \\
\hline HERV18 & ERV18 \\
\hline MER61 & ERVMER61 \\
\hline HERVH & $\mathrm{ERVH}$ \\
\hline HERV9 & ERV9 \\
\hline
\end{tabular}

information on the position of the ERVs within the genome or the order in which an ERV may have been published. The use of numerical identifiers keeps the symbols as short as possible to encourage widespread use by researchers. Newly identified transcribed loci will take the next available consecutive number for their particular group; for example, if a newly transcribed ERVK locus is identified, it will take the symbol ERVK-26. Each symbol is accompanied by an expanded gene name which clearly and succinctly explains that derivation of the nomenclature; for example, the full name of ERVFRD-1 is "endogenous retrovirus group FRD, member 1 ".

We are aware that the proposed nomenclature scheme cannot encompass all conceivable (and sometimes known) unusual structures of ERV loci, such as hybrid loci consisting of different ERV groups and ERV insertions into existing ERV loci [22]. HGNC, after conferring with researchers who submit newly identified transcribed loci, will decide whether or how to name such unique loci on a case-by-case basis. For example, the scheme will not incorporate ERV locus transcripts that are part of another gene's transcript, as these elements will not be considered separate loci.

Table 1 lists transcribed human ERVs that have been named according to the new nomenclature system. All ERVs in the table either have been published or have been annotated by the RefSeq project. An initial list was sent to a number of researchers in the field for their comments. The list was expanded as these researchers suggested more loci. Where no transcript sequence was available, authors were asked to submit representative sequences to the GenBank, EMBL and DDBJ databases. We encourage researchers to contact the HGNC if they know of further ERVs that can be included in the scheme.

Finally, although only human gene nomenclature is under the remit of the HGNC, we wish to mention that the naming system introduced here for transcribed ERVs could, in principle, also be applied to other, nonERV repetitive sequences in the human genome, as well as to repetitive DNA in nonhuman species. Future research will probably reveal numerous transcribed repetitive DNA sequences in various species. Judged just from ERV designations in different species, a standardised naming system for transcribed repeat loci may be highly beneficial to avoid future confusion.

\footnotetext{
Acknowledgements

Research in the laboratory of JM is supported by Deutsche Forschungsgemeinschaft (DFG) and Homburger

Forschungsförderungsprogramm (HOMFOR) The HGNC is funded by The Wellcome Trust grant 081979/Z/07/Z and National Human Genome Research Institute grant P41 HG03345). We also acknowledge those who provided extra information for the list of transcribed ERV loci: Yoshihiro
} 
Jinno, Finn Skou Pedersen, Benoit Barbeau, Christine Leib-Mösch and Patrick M Alliel.

\section{Author details}

'Department of Human Genetics, Theoretical Medicine and Biosciences, Centre for Human and Molecular Biology, Saarland University, D-66421 Homburg, Germany. ${ }^{2}$ Section of Virology, Department of Medical Sciences, Uppsala University, SE-751 85 Uppsala, Sweden. ${ }^{3} \mathrm{HUGO}$ Gene Nomenclature Committee, European Molecular Biology Laboratory-European Bioinformatics Institute, Wellcome Trust Genome Campus, Hinxton, Cambridgeshire CB10 1SD, UK.

\section{Authors' contributions}

All authors contributed to the presented nomenclature scheme and wrote the manuscript.

\section{Competing interests}

The authors declare that they have no competing interests.

Received: 11 February 2011 Accepted: 4 May 2011

Published: 4 May 2011

\section{References}

1. Lander ES, Linton LM, Birren B, Nusbaum C, Zody MC, Baldwin J, Devon K, Dewar K, Doyle M, FitzHugh W, et al: Initial sequencing and analysis of the human genome. Nature 2001, 409(6822):860-921.

2. Sperber GO, Airola T, Jern P, Blomberg J: Automated recognition of retroviral sequences in genomic data-RetroTector. Nucleic Acids Res 2007 35(15):4964-4976.

3. Blomberg J, Goran S, Jern P, Benachenhou F: Towards a retrovirus database, RetroBank. In Proceedings of the Centennial Retrovirus Meeting, 29 April - 4 May 2010. Edited by: Daniel R, Hejnar J, Skalka AM, Svoboda J Prague. Czech Republic: Medimond International Proceedings; 2010:19-22.

4. Mager DL, Medstrand P: Retroviral Repeat Sequences. In Nature Encyclopedia of the Human Genome. Volume 5. Edited by: Cooper D. Hampshire, England: Macmillan Publishers Ltd; 2003:57-63.

5. Gifford $R$, Tristem M: The evolution, distribution and diversity of endogenous retroviruses. Virus Genes 2003, 26(3):291-315.

6. Jurka J, Kapitonov W, Pavlicek A, Klonowski P, Kohany O, Walichiewicz J: Repbase Update, a database of eukaryotic repetitive elements. Cytogenet Genome Res 2005, 110(1-4):462-467.

7. Blikstad V, Benachenhou F, Sperber GO, Blomberg J: Evolution of human endogenous retroviral sequences: a conceptual account. Cell Mol Life Sci 2008, 65(21):3348-3365.

8. Kurth R, Bannert N: Beneficial and detrimental effects of human endogenous retroviruses. Int J Cancer 2010, 126(2):306-314.

9. Mayer J, Meese E: Human endogenous retroviruses in the primate lineage and their influence on host genomes. Cytogenet Genome Res 2005, 110(1-4):448-456

10. Ruprecht K, Mayer J, Sauter M, Roemer K, Mueller-Lantzsch N: Endogenous retroviruses and cancer. Cell Mol Life Sci 2008, 65(21):3366-3382.

11. Hu L: Endogenous retroviral RNA expression in humans. PhD thesis Department of Medical Sciences, Clinical Virology, Uppsala University; 2007.

12. Seifarth W, Frank O, Zeilfelder U, Spiess B, Greenwood AD, Hehlmann R, Leib-Mösch C: Comprehensive analysis of human endogenous retrovirus transcriptional activity in human tissues with a retrovirus-specific microarray. J Virol 2005, 79(1):341-352.

13. Mi S, Lee $X$, Li X, Veldman GM, Finnerty H, Racie L, LaVallie E, Tang XY Edouard $P$, Howes $S$, et al: Syncytin is a captive retroviral envelope protein involved in human placental morphogenesis. Nature 2000, 403(6771):785-789

14. de Parseval N, Heidmann T: Human endogenous retroviruses: from infectious elements to human genes. Cytogenet Genome Res 2005, 110(14):318-332.

15. Roebke C, Wahl S, Laufer G, Stadelmann C, Sauter M, Mueller-Lantzsch N, Mayer J, Ruprecht K: An N-terminally truncated envelope protein encoded by a human endogenous retrovirus $\mathrm{W}$ locus on chromosome Xq22.3. Retrovirology 2010, 7(1):69.

16. Blomberg J, Benachenhou F, Blikstad V, Sperber G, Mayer J: Classification and nomenclature of endogenous retroviral sequences (ERVs): problems and recommendations. Gene 2009, 448(2):115-123.
17. Seal RL, Gordon SM, Lush MJ, Wright MW, Bruford EA: genenames.org: the HGNC resources in 2011. Nucleic Acids Res 2011, 39 Database: D514-519.

18. Wain HM, Bruford EA, Lovering RC, Lush MJ, Wright MW, Povey S: Guidelines for human gene nomenclature. Genomics 2002, 79(4):464-470.

19. Birney E, Stamatoyannopoulos JA, Dutta A, Guigo R, Gingeras TR, Margulies EH, Weng Z, Snyder M, Dermitzakis ET, Thurman RE, et al: Identification and analysis of functional elements in 1\% of the human genome by the ENCODE pilot project. Nature 2007, 447(7146):799-816.

20. Mangan ME, Williams JM, Kuhn RM, Lathe WC: The UCSC genome browser: what every molecular biologist should know. Curr Protoc Mol Biol 2009, Chapter 19:Unit19 19.

21. Flicek P, Amode MR, Barrell D, Beal K, Brent S, Chen Y, Clapham P, Coates G, Fairley S, Fitzgerald S, et al: Ensembl 2011. Nucleic Acids Res 2011, , 39 Database: D800-806

22. Flockerzi A, Burkhardt S, Schempp W, Meese E, Mayer J: Human Endogenous Retrovirus HERV-K14 Families: Status, Variants, Evolution, and Mobilization of Other Cellular Sequences. J Virol 2005, 79(5):2941-2949.

23. Flockerzi A, Ruggieri A, Frank O, Sauter M, Maldener E, Kopper B, Wullich B, Seifarth W, Muller-Lantzsch N, Leib-Mosch C, et al: Expression patterns of transcribed human endogenous retrovirus HERV-K(HML-2) loci in human tissues and the need for a HERV Transcriptome Project. BMC Genomics 2008, 9:354.

24. Villesen P, Aagaard L, Wiuf C, Pedersen FS: Identification of endogenous retroviral reading frames in the human genome. Retrovirology 2004, 1:32

25. Sugimoto J, Matsuura N, Kinjo Y, Takasu N, Oda T, Jinno Y: Transcriptionally active HERV-K genes: identification, isolation, and chromosomal mapping. Genomics 2001, 72(2):137-144.

26. Berkhout $B$, Jebbink $M$, Zsiros J: Identification of an active reverse transcriptase enzyme encoded by a human endogenous HERV-K retrovirus. J Virol 1999, 73(3):2365-2375.

27. de Parseval N, Lazar V, Casella JF, Benit L, Heidmann T: Survey of human genes of retroviral origin: identification and transcriptome of the genes with coding capacity for complete envelope proteins. J Virol 2003, 77(19):10414-10422.

28. Barbulescu M, Turner G, Seaman MI, Deinard AS, Kidd KK, Lenz J: Many human endogenous retrovirus $\mathrm{K}$ (HERV-K) proviruses are unique to humans. Curr Biol 1999, 9(16):861-868.

29. Turner G, Barbulescu M, Su M, Jensen-Seaman MI, Kidd KK, Lenz J: Insertional polymorphisms of full-length endogenous retroviruses in humans. Curr Biol 2001, 11(19):1531-1535.

30. Zsiros J, Jebbink MF, Lukashov W, Voute PA, Berkhout B: Evolutionary relationships within a subgroup of HERV-K-related human endogenous retroviruses. J Gen Virol 1998, 79(Pt 1):61-70

31. Tonjes RR, Czauderna F, Kurth R: Genome-wide screening, cloning, chromosomal assignment, and expression of full-length human endogenous retrovirus type K. J Virol 1999, 73(11):9187-9195.

32. Okahara G, Matsubara S, Oda T, Sugimoto J, Jinno Y, Kanaya F: Expression analyses of human endogenous retroviruses (HERVs): tissue-specific and developmental stage-dependent expression of HERVs. Genomics 2004, 84(6):982-990

33. Frank O, Verbeke C, Schwarz N, Mayer J, Fabarius A, Hehlmann R, LeibMosch C, Seifarth W: Variable transcriptional activity of endogenous retroviruses in human breast cancer. J Virol 2008, 82(4):1808-1818.

34. Blaise $S$, de Parseval N, Benit L, Heidmann T: Genomewide screening for fusogenic human endogenous retrovirus envelopes identifies syncytin 2 a gene conserved on primate evolution. Proc Natl Acad Sci USA 2003, 100(22):13013-13018

35. Cohen $\mathrm{M}$, Powers $\mathrm{M}, \mathrm{O}^{\prime}$ Connell $\mathrm{C}$, Kato $\mathrm{N}$ : The nucleotide sequence of the env gene from the human provirus ERV3 and isolation and characterization of an ERV3-specific cDNA. Virology 1985, 147(2):449-458.

36. Larsson E, Andersson AC, Nilsson BO: Expression of an endogenous retrovirus (ERV3 HERV-R) in human reproductive and embryonic tissuesevidence for a function for envelope gene products. Ups J Med Sci 1994, 99(2):113-120

37. Blond $J$, Beseme F, Duret $L$, Bouton O, Bedin F, Perron H, Mandrand B, Mallet F: Molecular characterization and placental expression of HERV-W, a new human endogenous retrovirus family. J Virol 1999, 73(2):1175-1185.

38. Laufer G, Mayer J, Mueller BF, Mueller-Lantzsch N, Ruprecht K: Analysis of transcribed human endogenous retrovirus $\mathrm{W}$ env loci clarifies the origin 
of multiple sclerosis-associated retrovirus env sequences. Retrovirology 2009, 6:37.

39. Komurian-Pradel F, Paranhos-Baccala G, Bedin F, Ounanian-Paraz A, Sodoyer M, Ott C, Rajoharison A, Garcia E, Mallet F, Mandrand B, et al: Molecular cloning and characterization of MSRV-related sequences associated with retrovirus-like particles. Virology 1999, 260(1):1-9.

40. Jeong BH, Lee YJ, Carp Rl, Kim YS: The prevalence of human endogenous retroviruses in cerebrospinal fluids from patients with sporadic Creutzfeldt-Jakob disease. J Clin Virol 2010, 47(2):136-142.

41. Yao Y, Schroder J, Nellaker C, Bottmer C, Bachmann S, Yolken RH, Karlsson $\mathrm{H}$ : Elevated levels of human endogenous retrovirus-W transcripts in blood cells from patients with first episode schizophrenia. Genes Brain Behav 2008, 7(1):103-112.

42. de Parseval N, Diop G, Blaise S, Helle F, Vasilescu A, Matsuda F, Heidmann T: Comprehensive search for intra- and inter-specific sequence polymorphisms among coding envelope genes of retroviral origin found in the human genome: genes and pseudogenes. BMC Genomics 2005, 6:117.

43. Yi JM, Kim HS: Expression and phylogenetic analyses of human endogenous retrovirus HC2 belonging to the HERV-T family in human tissues and cancer cells. J Hum Genet 2007, 52(4):285-296.

44. Shiroma T, Sugimoto J, Oda T, Jinno Y, Kanaya F: Search for active endogenous retroviruses: identification and characterization of a HERV-E gene that is expressed in the pancreas and thyroid. J Hum Genet 2001, 46(11):619-625.

45. Prusty BK, zur Hausen H, Schmidt R, Kimmel R, de Villiers EM: Transcription of HERV-E and HERV-E-related sequences in malignant and nonmalignant human haematopoietic cells. Virology 2008, 382(1):37-45.

46. Takahashi Y, Harashima N, Kajigaya S, Yokoyama H, Cherkasova E, McCoy JP, Hanada K, Mena O, Kurlander R, Tawab A, et al: Regression of human kidney cancer following allogeneic stem cell transplantation is associated with recognition of an HERV-E antigen by T cells. J Clin Invest 2008, 118(3):1099-1109.

47. Kjeldbjerg AL, Villesen P, Aagaard L, Pedersen FS: Gene conversion and purifying selection of a placenta-specific ERV-V envelope gene during simian evolution. BMC Evol Biol 2008, 8:266.

48. Liang QY, Ding JY, Zheng S: Identification and detection of a novel human endogenous retrovirus-related gene, and structural characterization of its related elements. Genet Mol Biol 2009, 32(4):704-U738.

49. Patzke $S$, Lindeskog $M$, Munthe $E$, Aasheim HC: Characterization of a novel human endogenous retrovirus, $\mathrm{HERV}-\mathrm{H} / \mathrm{F}$, expressed in human leukemia cell lines. Virology 2002, 303(1):164-173.

50. Lindeskog M, Blomberg J: Spliced human endogenous retroviral HERV-H env transcripts in T-cell leukaemia cell lines and normal leukocytes: alternative splicing pattern of HERV-H transcripts. J Gen Virol 1997, 78(Pt 10):2575-2585.

51. Moles JP, Tesniere A, Guilhou JJ: A new endogenous retroviral sequence is expressed in skin of patients with psoriasis. Br J Dermatol 2005, 153(1):83-89.

52. La Mantia G, Pengue G, Maglione D, Pannuti A, Pascucci A, Lania L: Identification of new human repetitive sequences: characterization of the corresponding CDNAs and their expression in embryonal carcinoma cells. Nucleic Acids Res 1989, 17(15):5913-5922.

53. Andersson ML, Lindeskog M, Medstrand P, Westley B, May F, Blomberg J: Diversity of human endogenous retrovirus class II-like sequences. J Gen Virol 1999, 80(Pt 1):255-260.

doi:10.1186/1759-8753-2-7

Cite this article as: Mayer et al: A revised nomenclature for transcribed human endogenous retroviral loci. Mobile DNA 2011 2:7.

\section{Submit your next manuscript to BioMed Central and take full advantage of:}

- Convenient online submission

- Thorough peer review

- No space constraints or color figure charges

- Immediate publication on acceptance

- Inclusion in PubMed, CAS, Scopus and Google Scholar

- Research which is freely available for redistribution

Submit your manuscript at www.biomedcentral.com/submit
Biomed Central 